\title{
THE ESCAPING SET OF THE EXPONENTIAL
}

\author{
LASSE REMPE
}

\begin{abstract}
We show that the set $I(f)$ of points that converge to infinity under iteration of the exponential map $f(z)=\exp (z)$ is a connected subset of the complex plane.
\end{abstract}

\section{InTRODUCTION}

If $f: \mathbb{C} \rightarrow \mathbb{C}$ is an entire transcendental function, then its escaping set $I(f)$ is the set of points that tend to infinity under iteration:

$$
I(f)=\left\{z \in \mathbb{C}: f^{n}(z) \rightarrow \infty\right\} .
$$

For the dynamically simplest entire functions, such as exponential maps of the form $f(z)=\exp (z)+a$ with $a<-1$, the escaping set is the disjoint union of uncountably many curves to infinity, each of which is a connected component of $I(f)$. (In particular, $I(f)$ is disconnected while $I(f) \cup\{\infty\}$ is connected and path-connected.) Eremenko [E] conjectured that every connected component of $I(f)$ is unbounded for every transcendental entire function $f$. Despite recent progress (compare e.g. [R1, RS1, $\left.\mathrm{R}^{3} \mathrm{~S}\right]$ ), this question is still very much open.

In view of this, the escaping set is usually viewed very much as a set that is likely to be disconnected. However, Rippon and Stallard [RS1] proved that the escaping set of an entire function with a multiply-connected wandering domain is in fact connected. They have since extended this result to much larger classes of entire functions [RS2].

These examples are quite different from the exponential maps mentioned above in that they do not belong to the Eremenko-Lyubich class

$$
\mathcal{B}:=\left\{f: \mathbb{C} \rightarrow \mathbb{C} \text { transcendental, entire }: \operatorname{sing}\left(f^{-1}\right) \text { is bounded }\right\}
$$

(where $\operatorname{sing}\left(f^{-1}\right)$ denotes the set of critical and asymptotic values of $f$ ). We note that, if $f \in \mathcal{B}$, then $I(f)$ is a subset of the Julia set $J(f)$ [EL, Theorem 1]. Bergweiler (personal communication) asked whether the escaping set of a function in $\mathcal{B}$ can be connected, and more precisely whether this might be the case for the function $f(z)=\pi \sin (z)$. While Mihaljevic-Brandt [M-B] has given a negative answer to the latter, Rippon and Stallard observed that, for the function $f(z)=(\cosh (z))^{2}$, the escaping set is connected. Indeed, the union of the real axis with all its iterated preimages is path-connected (and clearly dense in $I(f))$.

In contrast to this example, for the exponential map $f(z)=\exp (z)$ every pathconnected component of the escaping set is known to be a single curve to $\infty$ that is relatively closed and nowhere dense in $I(f)$ (see Proposition 3.2). It may seem plausible

2000 Mathematics Subject Classification. Primary 37F10; Secondary 30D05,37F10,54F15.

Supported by EPSRC Fellowship EP/E052851/1. 
that these path-connected components are also the connected components of $I(f)$, but we show that the situation is rather different.

1.1. Theorem (Escaping set of the exponential). Let $a \in(-1, \infty)$ and consider the function $f(z)=\exp (z)+a$. Then $I(f)$ is a connected subset of the plane.

The proof is elementary; the main idea is to consider a countable sequence of preimage components of the negative real axis that was studied by Devaney [D] in his construction of an indecomposable continuum. (See Figure 1.) Each of these components is an arc tending to infinity in both directions, but we shall show that their union is connected. Theorem 1.1 then follows relatively easily.

Basic notation. As usual, we denote the complex plane by $\mathbb{C}$, and the Riemann sphere by $\hat{\mathbb{C}}=\mathbb{C} \cup\{\infty\}$. Closures and boundaries will be understood to be taken in $\mathbb{C}$, unless explicitly stated otherwise.

Acknowledgments. I would like to thank Walter Bergweiler, Mary Rees, Gwyneth Stallard and Phil Rippon for interesting discussions.

\section{The Devaney continuum}

For the rest of the article, fix $a \in(-1, \infty)$ and set $f(z)=\exp (z)+a$. Then $f^{n}(x) \rightarrow \infty$ for all $x \in \mathbb{R}$.

Let $\mathbb{H}_{+}$and $\mathbb{H}_{-}$denote the upper and lower half planes, respectively. Let $S_{+}$denote the strip at imaginary parts between 0 and $\pi$; similarly $S_{-}$is the strip at imaginary parts between 0 and $-\pi$. For $\sigma \in\{+,-\}$, let

$$
L_{\sigma}: \mathbb{H}_{\sigma} \rightarrow S_{\sigma}
$$

be the branch of $f^{-1}$ taking values in $S_{\sigma} . L_{\sigma}$ is a confomal isomorphism that extends to a homeomorphism between $\overline{\mathbb{H}_{\sigma}} \backslash\{a\}$ and $\overline{S_{\sigma}}$; we denote this extension also by $L_{\sigma}$.

Define $\gamma_{0}^{\sigma}:=(-\infty, a)$, and inductively

$$
\gamma_{k+1}^{\sigma}:=L_{\sigma}\left(\gamma_{k}^{\sigma}\right)
$$

Then each $\gamma_{k}^{\sigma}, k \geq 1$, is an injective curve tending to infinity in both directions. (Also, $\gamma_{k}^{-}$is the reflection of $\gamma_{k}^{+}$in the real axis for all $k$.)

We define sets $\Gamma^{\sigma}$ and $X^{\sigma}$ by

$$
\Gamma^{\sigma}:=\bigcup_{k \geq 0} \gamma_{k}^{\sigma}, \quad X^{\sigma}:=\overline{\Gamma^{\sigma}}
$$

See Figure 1 for a picture of the set $X^{+}$. We require the following key fact [D, p. 631]

2.1. Proposition (Hausdorff limit of $\gamma_{k}^{ \pm}$). Let $\sigma \in\{+,-\}$. The set $X^{\sigma} \cup\{\infty\}$ is the Hausdorff limit (on the Riemann sphere $\hat{\mathbb{C}}$ ) of the sequence $\left(\gamma_{k}^{\sigma} \cup\{\infty\}\right)$.

(In particular, $\bigcup_{k \geq k_{0}} \gamma_{k}^{\sigma}$ is dense in $X^{\sigma}$ for all $k_{0}$.)

Proof. Let $z_{0} \in X^{\sigma} \cup \infty$, and let $U$ be a neighborhood of $z_{0}$ in $\hat{\mathbb{C}}$. We need to show that $\gamma^{k} \cup U \neq \emptyset$ for all sufficiently large $k$.

By definition of $X^{\sigma}$, the set $U$ contains some $z_{1} \in \Gamma^{\sigma}$. Let $D_{n}$ denote the (Euclidean) disk of radius $2 \pi$ around $f^{n}\left(z_{1}\right)$. It is elementary to see - using the fact that $f$ is 
Figure 1. The set $X^{+}$

expanding in a suitable right half plane - that there is $n_{0}$ with $f^{j}(a) \notin D_{n}$ for all $n \geq n_{0}$ and $j<n$. We may assume that $n_{0}$ is chosen sufficiently large to ensure that also $f^{n}\left(z_{0}\right) \in \mathbb{R}$ for $n \geq n_{0}$.

Hence for $n \geq n_{0}$, there is a branch $\varphi_{n}: D_{n} \rightarrow \mathbb{C}$ of $f^{-n}$ with $\varphi_{n}\left(f^{n}\left(z_{0}\right)\right)=z_{0}$. Clearly $\max _{z \in D_{n}}\left|\varphi_{n}^{\prime}(z)\right| \rightarrow 0$ as $n \rightarrow \infty$ (again due to the expansion of $f$ in a right half plane). In particular, there is $k_{0} \geq n_{0}+1$ such that for $k \geq k_{0}$, the image of $\varphi_{k-1}$ is contained in $U$. We then have $\varphi_{k-1}\left(f^{k-1}\left(z_{0}\right)+\pi i\right) \in \gamma^{k} \cap U$, and the claim follows.

2.2. Proposition $\left(\Gamma^{ \pm}\right.$connected $)$. The sets $\Gamma^{+}$and $\Gamma^{-}$are connected.

Proof. Let $\sigma \in\{+,-\}$. Suppose that $U \subset \mathbb{C}$ is an open set with $\Gamma^{\sigma} \cap U \neq \emptyset$ and $\Gamma^{\sigma} \cap \partial U=\emptyset$. We need to show that $\Gamma^{\sigma} \subset U$.

Let $z_{0} \in U \cap \Gamma^{\sigma}$. Then by Proposition 2.1, there is $k_{0}$ such that $\gamma_{k} \cap U \neq \emptyset$ for all $k \geq k_{0}$. Since $\gamma_{k}$ is connected, in fact $\gamma_{k} \subset U$. Thus

$$
\Gamma^{\sigma} \subset X^{\sigma}=\overline{\bigcup_{k \geq k_{0}} \gamma_{k}^{\sigma}} \subset \bar{U} .
$$

By choice of $U$, we hence have $\Gamma^{\sigma} \subset U$, as desired.

\section{Proof of the Theorem}

By Proposition 2.2, the union of $\Gamma^{+}$and $\Gamma^{-}$connects the horizontal line $\gamma_{1}^{-}$at imaginary part $-\pi$ with $\gamma_{1}^{+}$at imaginary part $\pi$. Since the set $I(f)$ is $2 \pi i$-periodic, it follows that the set

$$
Y:=\bigcup_{\sigma \in\{+,-\}, k \in \mathbb{Z}}\left(\Gamma^{\sigma}+2 \pi i k\right)
$$

is a connected subset of $I(f) . \quad Y$ contains all points whose imaginary parts are odd multiples of $\pi$; i.e. $f^{-1}((-\infty, a))$.

3.1. Proposition (Preimages of $Y$ ). Set $Y_{0}:=Y$ and inductively $Y_{j+1}:=Y_{j} \cup f^{-1}\left(Y_{j}\right)$. Then $Y_{j}$ is connected for all $j$.

Proof. The proof is by induction on $j$. Note that $Y_{j}$ contains $Y$ for all $j$.

Let $k \in \mathbb{Z}$, and let $L_{k}: \mathbb{C} \backslash[a, \infty) \rightarrow \mathbb{C}$ be the branch of $f^{-1}$ that takes values with imaginary parts between $2 \pi k$ and $2 \pi(k+1)$. Set $z_{k}:=(2 k+1) \pi i$. Then $z_{k}, f\left(z_{k}\right) \in$ $Y \subset Y_{j}$, and hence $z_{k} \in Y_{j} \cap L_{k}\left(Y_{j}\right)$. As $L_{k}$ is a continuous function (and $Y_{j}$ is contained 
in its domain of definition), it follows from the induction hypothesis that $Y_{j} \cup L_{k}\left(Y_{j}\right)$ is connected. Hence

$$
Y_{j+1}=\bigcup_{k \in \mathbb{Z}} L_{k}\left(Y_{j}\right) \cup Y_{j}
$$

is connected, as claimed.

Proof of Theorem 1.1. The set $\bigcup_{j \geq 0} f^{-n}(-1) \subset \bigcup_{j \geq 0} Y_{j}$ is dense in the Julia set, and hence in the escaping set. Since $\bigcup_{j \geq 0} Y_{j}$ is a connected subset of $I(f)$, the claim follows.

We contrast our result with the following fact, mentioned in the introduction.

3.2. Proposition (Path-connected components of $I(f))$. Let $P$ be a path-connected component of $I(f)$, where again $f(z)=\exp (z)+a, a \in(-1, \infty)$. Then $P$ is relatively closed and nowhere dense in $I(f)$.

Proof. The path-connected components of $I(f)$ (in fact, of the escaping set of any exponential map) are completely described in [FRS, Corollary 4.3]. First of all, for any $n \geq 0$, each connected component of $f^{-n}(\mathbb{R})$ is a path-connected component of $I(f)$.

Every one of these is nowhere dense and closed in $\mathbb{C}$, and in particular relatively closed in $I(f)$.

Now suppose that $z_{0} \in I(f)$ never maps to the positive real axis under iteration. Let $\underline{s}=s_{0} s_{1} s_{2} \ldots$ be the sequence of integers such that $\operatorname{Im} f^{n}\left(z_{0}\right) \in\left(\left(2 s_{n}-1\right) \pi,\left(2 s_{n}+1\right) \pi\right)$ for all $n$. It follows from the assumption on $z_{0}$ that $\underline{s}$ must contain infinitely many nonzero entries (see [D, Theorem on p. 632]).

Let $K=K_{\underline{s}}$ be the set of all points $z \in J(f)$ with $\operatorname{Im} f^{n}(z) \in\left[\left(2 s_{n}-1\right) \pi,\left(2 s_{n}+1\right) \pi\right]$ for all $n \geq 0$. Clearly $K$ is closed and nowhere dense. It is known [DK, SZ] that $K \cap I(f)$ is path-connected; in fact, $K \cap I(f)$ is the trace of an injective curve $g_{\underline{s}}:[0, \infty) \rightarrow \mathbb{C}$ or $g_{\underline{s}}:(0, \infty) \rightarrow \mathbb{C}$ with $g_{\underline{s}}(t) \rightarrow \infty$ as $t \rightarrow \infty$. (This curve is called a Devaney hair or a dynamic ray.) We remark that, for certain addresses $\underline{s}$, the limit set of $g_{\underline{s}}(t)$ as $t \rightarrow 0$ will not consist of a single point [DJ] (compare also [R2]). Any escaping points in this limit set must necessarily lie on $g_{\underline{s}}$ themselves.

By [FRS, Corollary 4.3], the curve $P:=K \cap I(f)=g_{\underline{s}}$ is the path-connected component of $I(f)$ containing $z_{0}$. Since $K$ is closed and nowhere dense, the claim follows.

\section{REFERENCES}

[D] Robert L. Devaney, Knaster-like continua and complex dynamics, Ergodic Theory Dynam. Systems 13 (1993), no. 4, 627-634.

[DJ] Robert L. Devaney and Xavier Jarque, Indecomposable continua in exponential dynamics, Conform. Geom. Dyn. 6 (2002), 1-12.

[DK] Robert L. Devaney and Michał Krych, Dynamics of $\exp (z)$, Ergodic Theory Dynam. Systems 4 (1984), no. 1, 35-52.

[E] Alexandre È. Eremenko, On the iteration of entire functions, Dynamical systems and ergodic theory (Warsaw, 1986), Banach Center Publ., vol. 23, PWN, Warsaw, 1989, pp. 339-345.

[EL] Alexandre È. Eremenko and Mikhail Yu. Lyubich, Dynamical properties of some classes of entire functions, Ann. Inst. Fourier (Grenoble) 42 (1992), no. 4, 989-1020.

[FRS] Markus Förster, Lasse Rempe, and Dierk Schleicher, Classification of escaping exponential maps, Preprint, 2004, arXiv:math.DS/0311427, to appear in Proc. Amer. Math. Soc.

[M-B] Helena Mihaljević-Brandt, Orbifolds of subhyperbolic transcendental maps, Manuscript, 2008. 
[R1] Lasse Rempe, On a question of Eremenko concerning escaping sets of entire functions, Bull. London Math. Soc. 39 (2007), no. 4, 661-666, arXiv:math.DS/0610453.

[R2] - On nonlanding dynamic rays of exponential maps, Ann. Acad. Sci. Fenn. Math. 32 (2007), 353-369, arXiv:math.DS/0511588

[RS1] Philip J. Rippon and Gwyneth M. Stallard, On questions of Fatou and Eremenko, Proc. Amer. Math. Soc. 133 (2005), no. 4, 1119-1126.

[RS2] _ Escaping points of entire functions of small growth, Math. Z. (to appear), arXiv:0801.3605.

$\left[\mathrm{R}^{3} \mathrm{~S}\right]$ Günter Rottenfußer, Johannes Rückert, Lasse Rempe, and Dierk Schleicher, Dynamic rays of entire functions, Preprint \#2007/05, Institute for Mathematical Sciences, SUNY Stony Brook, 2007, arXiv:0704.3213, submitted for publication.

[SZ] Dierk Schleicher and Johannes Zimmer, Escaping points of exponential maps, J. London Math. Soc. (2) 67 (2003), no. 2, 380-400.

Dept. of Mathematical Sciences, University of Liverpool, Liverpool L69 7ZL, UK

E-mail address: 1.rempe@liverpool.ac.uk 